TRANSACTIONS OF THE

AMERICAN MATHEMATICAL SOCIETY

Volume 364, Number 5, May 2012, Pages 2241-2260

S 0002-9947(2012)05598-X

Article electronically published on January 19, 2012

\title{
ON RESTRICTION OF MAXIMAL MULTIPLIERS IN WEIGHTED SETTINGS
}

\author{
MARÍA J. CARRO AND SALVADOR RODRÍGUEZ-LÓPEZ
}

\begin{abstract}
We obtain restriction results of K. De Leeuw's type for maximal operators defined through Fourier multipliers of either strong or weak type for weighted $L^{p}$ spaces with $1 \leq p<\infty$. Applications to the case of HörmanderMihlin multipliers, singular integral operators and Bochner-Riesz sums are given.
\end{abstract}

\section{INTRODUCTION}

In $1965 \mathrm{~K}$. De Leeuw proved that if $\mathbf{m}$ is a continuous function on $\mathbb{R}$ such that $\mathbf{m}$ is a Fourier multiplier on $L^{p}(\mathbb{R})$, its restriction to the integers $\left.\mathbf{m}\right|_{\mathbb{Z}}$ is a Fourier multiplier on $L^{p}(\mathbb{T})$. Moreover, its norm does not exceed the norm of $\mathbf{m}$ as a multiplier on $L^{p}(\mathbb{R})$ (see [8, Proposition 3.3] and Jodeit's article [12]).

In 1980 C. Kenig and P. Tomas extended De Leeuw's result to maximal operators associated to a family of multipliers given by the dilations of a given one. More precisely, they proved that if $\mathbf{m}$ is a continuous function and if $T_{r}$ denotes the multiplier operator associated to $\mathbf{m}_{r}(\xi)=\mathbf{m}(\xi / r)$, whenever $T^{\sharp} f(x)=\sup _{r>0}\left|T_{r} f(x)\right|$ is a bounded operator on $L^{p}\left(\mathbb{R}^{d}\right)$ the same holds for the maximal operator on $L^{p}\left(\mathbb{T}^{d}\right)$ associated to the multipliers $\left.\mathbf{m}_{r}\right|_{\mathbb{Z}}$. Furthermore, its norm does not exceed a constant times the norm of $T^{\sharp}$. They also obtained similar results for operators of weak type for $p>1$ (see [13).

In 2003, E. Berkson and T.A. Gillespie extended De Leeuw's restriction result for multipliers on $L^{p}(\mathbb{R}, w)$ with $w$ a 1 -periodic weight belonging to $A_{p}(\mathbb{R})$ and $1<p<\infty$. Such weights are said to be in the class $A_{p}(\mathbb{T})$. Their result is the following.

Theorem 1.1 ([4, Theorem 1.2]). Let $1<p<\infty$ and let $w \in A_{p}(\mathbb{T})$. If $\mathbf{m}$ is a continuous function on $\mathbb{R}$ such that it is a Fourier multiplier for $L^{p}(\mathbb{R}, w)$, then $\left.\mathbf{m}\right|_{\mathbb{Z}}$ is a Fourier multiplier on $L^{p}(\mathbb{T}, w)$. Moreover, there is a constant $\mathfrak{c}_{p, w}$ depending only on $p$ and the $A_{p}$-constant of $w$, such that the norm of $\left.\mathbf{m}\right|_{\mathbb{Z}}$ as a multiplier on $L^{p}(\mathbb{T}, w)$ does not exceed $\mathfrak{c}_{p, w}$ times the norm of $\mathbf{m}$ as a multiplier on $L^{p}(\mathbb{R}, w)$.

This theorem has been recently improved by K. Andersen and P. Mohanty as follows.

Theorem 1.2 ([1, Theorem 1.1]). Let $1<p<\infty$ and let $w \in L^{1}\left(\mathbb{T}^{d}\right)$. If $\mathbf{m}$ is a continuous function on $\mathbb{R}^{d}$ such that it is a Fourier multiplier on $L^{p}\left(\mathbb{R}^{d}, w\right)$,

Received by the editors July 13, 2009.

2010 Mathematics Subject Classification. Primary 42B15, 42B35.

Key words and phrases. Weighted $L^{p}$ spaces, Fourier multipliers.

This work was partially supported by MTM2010-14946. The second author was also partially supported by EP/H051368/1. 
then $\left.\mathbf{m}\right|_{\mathbb{Z}}$ is a Fourier multiplier on $L^{p}\left(\mathbb{T}^{d}, w\right)$. Moreover, the norm of $\left.\mathbf{m}\right|_{\mathbb{Z}}$ as a multiplier on $L^{p}\left(\mathbb{T}^{d}, w\right)$ does not exceed the norm of $\mathbf{m}$ as a multiplier on $L^{p}\left(\mathbb{R}^{d}, w\right)$.

The purpose of this paper is twofold:

i) To give restriction results from $\mathbb{R}^{d}$ to $\mathbb{T}^{d}$ for Fourier multipliers and for associated maximal operators of weak type (and strong type) in any dimension and for $1 \leq p<\infty$. In particular, we shall prove the following.

Theorem 1.3. Let $1 \leq p<\infty$ and let $w$ be a periodic weight on $\mathbb{R}^{d}$ satisfying $w \in$ $L^{1}\left(\mathbb{T}^{d}\right)$. Suppose that $\left\{\mathbf{m}_{j}\right\}_{j}$ is a family of multipliers that are continuous functions satisfying that the associated maximal operator (see Definition 2.2 below) is bounded from $L^{p}\left(\mathbb{R}^{d}, w\right)$ to $L^{p, \infty}\left(\mathbb{R}^{d}, w\right)$ (or to $L^{p}\left(\mathbb{R}^{d}, w\right)$ ). Then the maximal operator associated to their restriction to the integers $\left\{\left.\mathbf{m}_{j}\right|_{\mathbb{Z}^{d}}\right\}_{j}$ (see Definition 3.1 below) is bounded from $L^{p}\left(\mathbb{T}^{d}, w\right)$ to $L^{p, \infty}\left(\mathbb{T}^{d}, w\right)$ (resp. to $L^{p}\left(\mathbb{T}^{d}, w\right)$ ) and its operator norm does not exceed $c_{p}$ times the norm of the maximal operator associated to $\left\{\mathbf{m}_{j}\right\}_{j}$, where $c_{p}$ is a constant that depends only on $p$.

ii) K. De Leeuw in [8] and J. Jodeit in [12] also proved some restriction results for strong Fourier multipliers on $L^{p}\left(\mathbb{R}^{d}\right)$ to a lower dimensional space. In [7] a counterpart for Fourier multipliers on $L^{p}\left(\mathbb{R}^{d}, w\right)$ with $w$ a suitable weight in $A_{p}\left(\mathbb{R}^{d}\right)$ was given. Namely, [7, Corollary 4.13] states that if $\mathbf{m}$ is a continuous and bounded function in $\mathbb{R}^{d}$ that is a Fourier multiplier on $L^{p}\left(\mathbb{R}^{d}, w\right)$ where $w=u \otimes v$ with $u \in A_{p}\left(\mathbb{R}^{d_{1}}\right), v \in A_{p}\left(\mathbb{R}^{d_{2}}\right)$, then, for any $\xi \in \mathbb{R}^{d_{1}}$, the function $\mathbf{m}(\xi, \cdot)$ is a Fourier multiplier on $L^{p}\left(\mathbb{R}^{d_{2}}, v\right)$. In this setting, we shall prove the following.

Theorem 1.4. Let $d=d_{1}+d_{2}, 1 \leq p<\infty, u \in A_{p}\left(\mathbb{R}^{d_{1}}\right), v \in A_{p}\left(\mathbb{R}^{d_{2}}\right)$ and define $w(x, y)=u(x) v(y)$. Suppose that $\left\{\mathbf{m}_{j}\right\}_{j}$ is a family of multipliers that are continuous functions satisfying that the associated maximal operator is bounded from $L^{p}\left(\mathbb{R}^{d}, w\right)$ to $L^{p, \infty}\left(\mathbb{R}^{d}, w\right)$ (or to $L^{p}\left(\mathbb{R}^{d}, w\right)$ ). Then, fixed $\xi \in \mathbb{R}^{d_{1}}$, the maximal operator associated to the family $\left\{\mathbf{m}_{j}(\xi, \cdot)\right\}_{j}$ is bounded from $L^{p}\left(\mathbb{R}^{d_{2}}, v\right)$ to $L^{p, \infty}\left(\mathbb{R}^{d_{2}}, v\right)$ (resp. to $\left.L^{p}\left(\mathbb{R}^{d_{2}}, v\right)\right)$ and its operator norm does not exceed $\mathfrak{c}_{p, w}$ times the norm of the maximal operator associated to $\left\{\mathbf{m}_{j}\right\}_{j}$, where $\mathfrak{c}_{p, w}$ is a constant that depends only on $p, d$ and the $A_{p}$-constant of $w$.

We want to emphasize that the techniques developed in this paper are different from those in [1,4,7, where duality properties of Lebesgue spaces are strongly used. Our approach allows us to also consider the case of maximal multipliers of weak type $(1,1)$, and deal with the difficulties derived from the fact that $L^{1, \infty}$ is not a Banach space. The endpoint case $p=1$ is the weighted analogue of the results in 2, 15.

\section{Definitions and notation}

In this section we present some basic definitions needed for our consideration. Let $0<p<\infty$ and let $(\mathcal{M}, \mu)$ be a $\sigma$-finite measure space. The space $L^{p, \infty}(\mu)$ is defined by the quasinorm $\|f\|_{L^{p, \infty}}=\sup _{t>0} t \mu_{f}(s)^{1 / p}$, where $\mu_{f}(s)=\mu\{x:|f(x)|>s\}$. It is known (see [10, p. 485]) that, for every $q<p$,

$$
\|f\|_{L^{p, \infty}(\mu)} \leq \sup \left\|f \chi_{E}\right\|_{L^{q}(\mu)} \mu(E)^{1 / p-1 / q} \leq c_{p, q}\|f\|_{L^{p, \infty}(\mu)},
$$

where the supremum is taken on the family of sets of finite measure and $c_{p, q}^{q}=\frac{p}{p-q}$. The finiteness of the middle expression is called Kolmogorov's condition. 
If $\nu$ is a positive measure absolutely continuous with respect to $\mu$ and $w$ denotes the Radon-Nykodym derivative of $\nu$ with respect to $\mu$, we shall write $L^{p}(w)$ for $L^{p}(\nu)$. If any confusion can arise, we shall write $L^{p}(\mathcal{M}, \mu)$ and $L^{p, \infty}(\mathcal{M}, \mu)$ to indicate the underlying measure space $\mathcal{M}$.

Let $\mathcal{C}_{c}^{\infty}\left(\mathbb{R}^{d}\right)$ and $\mathcal{S}\left(\mathbb{R}^{d}\right)$ denote the class of infinitely differentiable functions with compact support and the Schwartz class of test functions, respectively. As usual, $\mathfrak{B}(X, Y)$ indicates the set of bounded operators on $X$ into $Y$ and $\mathfrak{B}(X)=\mathfrak{B}(X, X)$.

A weight on $\mathbb{R}^{d}$ is a locally integrable function $w: \mathbb{R}^{d} \rightarrow[0, \infty)$ such that $0<w<\infty$ a.e.

Definition 2.1. We say that a weight $w$ belongs to the class $A_{p}\left(\mathbb{R}^{d}\right)$, and we write $w \in A_{p}\left(\mathbb{R}^{d}\right)$ if

$$
[w]_{A_{p}}=\sup _{Q}\left(\frac{1}{|Q|} \int_{Q} w(x) d x\right)\left(\frac{1}{|Q|} \int_{Q} w(x)^{1 / 1-p} d x\right)^{p-1}<\infty
$$

for $1<p<\infty$, and

$$
[w]_{A_{1}}=\sup _{Q}\left(\frac{1}{|Q|} \int_{Q} w(x) d x\right)\left\|w^{-1} \chi_{Q}\right\|_{\infty}<+\infty
$$

where the supremum is taken over the family of cubes $Q$ with sides parallel to the coordinate axis. These quantities will be referred to as the $A_{p}$-constant of $w$.

It is well known that, for $1 \leq p<\infty$ and $w \in A_{p}\left(\mathbb{R}^{d}\right), \mathcal{S}\left(\mathbb{R}^{d}\right) \subset L^{p}\left(\mathbb{R}^{d}, w\right)$ and $\mathcal{C}_{c}^{\infty}\left(\mathbb{R}^{d}\right)$ is dense in $L^{p}\left(\mathbb{R}^{d}, w\right)$. We refer the reader to [10,11] for other properties and generalities of $A_{p}$-weights.

For any function $f$, we shall denote by $\widehat{f}\left(f^{\vee}\right)$ the Fourier transform (resp. the inverse Fourier transform) of $f$, whenever it is well defined.

Definition 2.2. Let $1 \leq p<\infty$. A function $\mathbf{m} \in L^{\infty}\left(\mathbb{R}^{d}\right)$ is called a weak type multiplier on $L^{p}\left(\mathbb{R}^{d}, w\right)$ (in symbols, $\mathbf{m} \in M_{p, w}^{(\mathfrak{w})}\left(\mathbb{R}^{d}\right)$ ) if the mapping $f \in$ $\mathcal{S}\left(\mathbb{R}^{d}\right) \mapsto(\mathbf{m} \hat{f})^{\vee}$ can be extended from $\mathcal{S}\left(\mathbb{R}^{d}\right)$ to a continuous linear mapping $S_{\mathbf{m}}$ from $L^{p}\left(\mathbb{R}^{d}, w\right)$ to $L^{p, \infty}\left(\mathbb{R}^{d}, w\right)$. In this case we write

$$
\|\mathbf{m}\|_{M_{p, w}^{(\mathfrak{w})}\left(\mathbb{R}^{d}\right)}=\left\|S_{\mathbf{m}}\right\|_{\mathfrak{B}\left(L^{p}\left(\mathbb{R}^{d}, w\right), L^{p, \infty}\left(\mathbb{R}^{d}, w\right)\right)} .
$$

If $S_{\mathbf{m}} \in \mathfrak{B}\left(L^{p}\left(\mathbb{R}^{d}, w\right)\right)$, we say that $\mathbf{m}$ is a Fourier multiplier on $L^{p}\left(\mathbb{R}^{d}, w\right)$ and write

$$
\|\mathbf{m}\|_{M_{p, w}\left(\mathbb{R}^{d}\right)}=\left\|S_{\mathbf{m}}\right\|_{\mathfrak{B}\left(L^{p}\left(\mathbb{R}^{d}, w\right)\right)} .
$$

If $\left\{\mathbf{m}_{j}\right\}_{j}$ is a sequence in $M_{p, w}^{(\mathfrak{w})}\left(\mathbb{R}^{d}\right)$, we denote by $\left\|\left\{\mathbf{m}_{j}\right\}_{j}\right\|_{M_{p, w}^{(\mathfrak{w})}\left(\mathbb{R}^{d}\right)}$ the norm of the operator defined for $f \in \mathcal{S}\left(\mathbb{R}^{d}\right)$ by

$$
S_{\left\{\mathbf{m}_{j}\right\}_{j}}^{\sharp} f(x)=\sup _{j}\left|S_{\mathbf{m}_{j}} f(x)\right|,
$$

provided it defines a continuous mapping from $L^{p}\left(\mathbb{R}^{d}, w\right)$ to $L^{p, \infty}\left(\mathbb{R}^{d}, w\right)$. If it extends to a bounded mapping on $L^{p}\left(\mathbb{R}^{d}, w\right)$, we write its norm by $\left\|\left\{\mathbf{m}_{j}\right\}_{j}\right\|_{M_{p, w}\left(\mathbb{R}^{d}\right)}$.

We shall denote by $\mathbb{T}^{d}$ the topological group $\mathbb{R}^{d} / \mathbb{Z}^{d}$, which can be identified with the cube $[0,1)^{d}$ or eventually with $[-1 / 2,1 / 2)^{d}$ in $\mathbb{R}^{d}$. Functions on $\mathbb{T}^{d}$ will be identified with functions on $\mathbb{R}^{d}$ which are 1-periodic in each variable. A function 
$f: \mathbb{T}^{d} \rightarrow \mathbb{C}$ such that for a finitely supported sequence $\left\{a_{k}\right\}_{k \in \mathbb{Z}^{d}}$ of complex numbers written as

$$
f(x)=\sum_{k \in \mathbb{Z}^{d}} a_{k} e^{2 \pi i k x}
$$

is called a trigonometric polynomial, and we write $f \in P\left(\mathbb{T}^{d}\right)$. Let us recall that $P\left(\mathbb{T}^{d}\right)$ is dense in $L^{p}\left(\mathbb{T}^{d}, \mu\right)$ for any Radon measure $\mu$ on $\mathbb{T}^{d}$.

From now on, we work in the range

$$
1 \leq p<\infty
$$

and $w$ is a weight in $\mathbb{R}^{d}$. Observe that if in addition $w$ is 1-periodic, then $w \in$ $L^{1}\left(\mathbb{T}^{d}\right)$.

\section{Restriction of Fourier multipliers from $\mathbb{R}^{d}$ TO $\mathbb{T}^{d}$}

Definition 3.1. A function $\mathbf{m} \in \ell^{\infty}\left(\mathbb{Z}^{d}\right)$ is a weak type multiplier on $L^{p}\left(\mathbb{T}^{d}, w\right)$ (in symbols, $\mathbf{m} \in M_{p, w}^{(\mathfrak{w})}\left(\mathbb{T}^{d}\right)$ ) if the mapping

$$
\sum_{k \in \mathbb{Z}^{d}} a_{k} e^{2 \pi i k \theta} \in P\left(\mathbb{T}^{d}\right) \longrightarrow \sum_{k \in \mathbb{Z}^{d}} \mathbf{m}(k) a_{k} e^{2 \pi i k \theta}
$$

extends to a continuous operator $T_{\mathbf{m}} \in \mathfrak{B}\left(L^{p}\left(\mathbb{T}^{d}, w\right), L^{p, \infty}\left(\mathbb{T}^{d}, w\right)\right)$. In this case,

$$
\|\mathbf{m}\|_{M_{p, w}^{(\mathfrak{w})}\left(\mathbb{T}^{d}\right)}=\left\|T_{\mathbf{m}}\right\|_{\mathfrak{B}\left(L^{p}\left(\mathbb{T}^{d}, w\right), L^{p, \infty}\left(\mathbb{T}^{d}, w\right)\right)} .
$$

If $T_{\mathbf{m}} \in \mathfrak{B}\left(L^{p}\left(\mathbb{T}^{d}, w\right)\right), \mathbf{m}$ is said to be a multiplier on $L^{p}\left(\mathbb{T}^{d}, w\right)$, we denote it by $\mathbf{m} \in M_{p, w}\left(\mathbb{T}^{d}\right)$ and

$$
\|\mathbf{m}\|_{M_{p, w}\left(\mathbb{T}^{d}\right)}=\left\|T_{\mathbf{m}}\right\|_{\mathfrak{B}\left(L^{p}\left(\mathbb{T}^{d}, w\right)\right)} .
$$

If $\left\{\mathbf{m}_{j}\right\}_{j}$ is a sequence in $M_{p, w}^{(\mathfrak{w})}\left(\mathbb{T}^{d}\right)$ we denote by $\left\|\left\{\mathbf{m}_{j}\right\}_{j}\right\|_{M_{p, w}^{(\mathfrak{w})}\left(\mathbb{T}^{d}\right)}$ the norm of the operator defined for every $f \in P\left(\mathbb{T}^{d}\right)$ by

$$
T_{\left\{\mathbf{m}_{j}\right\}_{j}}^{\sharp} f(x)=\sup _{j}\left|T_{\mathbf{m}_{j}} f(x)\right|,
$$

provided it extends to a continuous mapping from $L^{p}\left(\mathbb{T}^{d}, w\right)$ to $L^{p, \infty}\left(\mathbb{T}^{d}, w\right)$. We shall write $\left\|\left\{\mathbf{m}_{j}\right\}_{j}\right\|_{M_{p, w}\left(\mathbb{T}^{d}\right)}$ in the case that it extends to a continuous operator on $L^{p}\left(\mathbb{T}^{d}, w\right)$.

\subsection{Restriction results for weak type maximal multipliers.}

Theorem 3.2. Let $w$ be 1-periodic and let $\left\{\mathbf{m}_{j}\right\}_{j} \in M_{p, w}^{(\mathfrak{w})}\left(\mathbb{R}^{d}\right)$ satisfying that, for each $j$, there exists $K_{j} \in L^{1}\left(\mathbb{R}^{d}\right)$ with compact support such that $\hat{K}_{j}(x)=\mathbf{m}_{j}(x)$ for every $x \in \mathbb{R}^{d}$. Then $\left\{\left.\mathbf{m}_{j}\right|_{\mathbb{Z}^{d}}\right\}_{j} \in M_{p, w}^{(\mathfrak{w})}\left(\mathbb{T}^{d}\right)$ and

$$
\left\|\left\{\left.\mathbf{m}_{j}\right|_{\mathbb{Z}^{d}}\right\}_{j}\right\|_{M_{p, w}^{(\mathfrak{w})}\left(\mathbb{T}^{d}\right)} \leq c_{p}\left\|\left\{\mathbf{m}_{j}\right\}_{j}\right\|_{M_{p, w}^{(\mathfrak{w})}\left(\mathbb{R}^{d}\right)},
$$

where $c_{p}$ depends only on $p$.

Proof. Let $\mathfrak{N}=\left\|\left\{\mathbf{m}_{j}\right\}_{j}\right\|_{M_{p, w}^{(\mathfrak{w})}\left(\mathbb{R}^{d}\right)}$. Since convolution operators commute with translations, it follows that for every $\theta \in[0,1)^{d}$ and every $N \in \mathbb{N}$,

$$
\left\|\sup _{1 \leq j \leq N}\left|K_{j} * g\right|\right\|_{L^{p, \infty}\left(\mathbb{R}^{d}, w(\cdot+\theta)\right)} \leq \mathfrak{N}\|g\|_{L^{p}\left(\mathbb{R}^{d}, w(\cdot+\theta)\right)} .
$$


Now, given $f(\theta)=\sum_{k} a_{k} e^{2 \pi i k \theta} \in P\left(\mathbb{T}^{d}\right)$, let us consider

$$
\begin{aligned}
\tilde{T}_{K_{j}} f(\theta) & =\int_{\mathbb{R}^{d}} K_{j}(x) f(\theta-x) d x=\sum_{k} a_{k} \int_{\mathbb{R}^{d}} K_{j}(x) e^{2 \pi i k(\theta-x)} d x \\
& =\sum_{k \in \mathbb{Z}^{d}} a_{k} \mathbf{m}_{j}(k) e^{2 \pi i k \theta}
\end{aligned}
$$

that is, $\tilde{T}_{K_{j}}$ coincides with the multiplier operator $T_{\left.\mathbf{m}_{j}\right|_{I^{d}}}$.

Let $Q_{r}=(-r, r)^{d}$ with $r>0$ such that $\operatorname{supp} K_{j} \subset Q_{r}$ for $j=1, \ldots, N$. Let $q<p$, and for any measurable $E \subset[0,1)^{d}$, let $\tilde{E}=\bigcup_{k \in \mathbb{Z}^{d}} E+k$ be its periodic extension. Set $E_{\theta}=\left\{x \in \mathbb{R}^{d}: x+\theta \in \tilde{E}\right\}$ with $\theta \in \mathbb{T}^{d}$ and $R_{x} f(\theta)=f(\theta+x)$. Then, by translation invariance, we have that, for every $x \in \mathbb{R}^{d}$,

$$
\left\|\sup _{1 \leq j \leq N}\left|\tilde{T}_{K_{j}} f\right| \chi_{E}\right\|_{L^{q}\left(\mathbb{T}^{d}, w\right)}^{q}=\int_{\mathbb{T}^{d}} \sup _{1 \leq j \leq N}\left|R_{x} \tilde{T}_{K_{j}} f(\theta)\right|^{q} w(x+\theta) \chi_{\tilde{E}}(x+\theta) d \theta
$$

Therefore, for every $s>0$,

$$
\begin{aligned}
& \left\|\sup _{1 \leq j \leq N}\left|\tilde{T}_{K_{j}} f\right| \chi_{E}\right\|_{L^{q}\left(\mathbb{T}^{d}, w\right)}^{q} \\
& \quad=\frac{1}{(2 s)^{d}} \int_{Q_{s}} \int_{\mathbb{T}^{d}} \sup _{1 \leq j \leq N}\left|R_{x} T_{K_{j}} f(\theta)\right|^{q} w(x+\theta) \chi_{\tilde{E}}(x+\theta) d \theta d x .
\end{aligned}
$$

Now, using that $\operatorname{supp} K_{j} \subset Q_{r}$ for $j=1, \ldots, N$, one can easily see that, if $x \in Q_{s}$,

$$
R_{x} \tilde{T}_{K_{j}} f(\theta)=B_{K_{j}}\left(R_{(\cdot)} f(\theta) \chi_{Q_{r+s}}\right)(x),
$$

where $B_{K_{j}}(h)(x)=\left(K_{j} * h\right)(x)$, and hence,

$$
\begin{aligned}
& \left\|\sup _{1 \leq j \leq N}\left|\tilde{T}_{K_{j}} f\right| \chi_{E}\right\|_{L^{q}\left(\mathbb{T}^{d}, w\right)}^{q} \\
& \quad \leq \frac{1}{(2 s)^{d}} \int_{\mathbb{T}^{d}}\left\{\int_{E_{\theta} \cap Q_{s}} \sup _{1 \leq j \leq N}\left|B_{K_{j}}\left(R_{(\cdot)} f(\theta) \chi_{Q_{r+s}}\right)(x)\right|^{q} w(x+\theta) d x\right\} d \theta .
\end{aligned}
$$

By (2.1) and (3.1), the term inside curly brackets is bounded by

$$
\left(c_{p, q} \mathfrak{N}\right)^{q}\left\{\int_{Q_{r+s}}\left|R_{x} f(\theta)\right|^{p} w(x+\theta) d x\right\}^{\frac{q}{p}}\left\{\int_{E_{\theta} \cap Q_{s}} w(x+\theta) d x\right\}^{1-\frac{q}{p}} .
$$

Also, using Hölder's inequality, it follows that

$$
\begin{aligned}
& \left\|\sup _{1 \leq j \leq N}\left|\tilde{T}_{K_{j}} f\right| \chi_{E}\right\|_{L^{q}\left(\mathbb{T}^{d}, w\right)}^{q} \\
& \leq \frac{c_{p, q}^{q} \mathfrak{N}^{q}}{(2 s)^{d}}\left\{\int_{\mathbb{T}^{d}} \int_{Q_{r+s}}\left|R_{x} f(\theta)\right|^{p} w(x+\theta) d t d \theta\right\}^{\frac{q}{p}}\left\{\int_{\mathbb{T}^{d}} \int_{Q_{s} \cap E_{\theta}} w(x+\theta) d x d \theta\right\}^{1-\frac{q}{p}} \\
& \leq \frac{c_{p, q}^{q} \mathfrak{N}^{q}}{(2 s)^{d}}(2(r+s))^{\frac{d q}{p}}(2 s)^{d\left(1-\frac{q}{p}\right)} w(E)^{1-\frac{q}{p}}\|f\|_{L^{p}\left(\mathbb{T}^{d}, w\right)}^{q} \\
& \leq c_{p, q}^{q} \mathfrak{N}^{q}\left(\frac{r+s}{s}\right)^{\frac{d q}{q}} w(E)^{1-\frac{q}{p}}\|f\|_{L^{p}\left(\mathbb{T}^{d}, w\right)}^{q} .
\end{aligned}
$$


Thus, taking $s \rightarrow+\infty$, and using Kolmogorov's condition (2.1), we obtain that

$$
\left\|\sup _{1 \leq j \leq N}\left|\tilde{T}_{K_{j}} f\right|\right\|_{L^{p, \infty\left(\mathbb{T}^{d}, w\right)}} \leq c_{p, q} \mathfrak{N}\|f\|_{L^{p}\left(\mathbb{T}^{d}, w\right)}
$$

Now, considering $c_{p}=\inf _{q<p} c_{p, q}$, the result easily follows by Fatou's Lemma and the density of $P\left(\mathbb{T}^{d}\right)$ in $L^{p}\left(\mathbb{T}^{d}, w\right)$.

The next step is to weaken the hypothesis assumed on $\mathbf{m}_{j}$ in the previous theorem as is done both in [4 and 1. As usually happens, this is the technical part of the work.

Definition 3.3. A bounded function $\mathbf{m}$ defined in $\mathbb{R}^{d}$ is normalized if for any $x \in \mathbb{R}^{d}$,

$$
\lim _{n} \widehat{\varphi_{n}} * \mathbf{m}(x)=\mathbf{m}(x),
$$

where $\varphi_{n}(x)=\varphi(x / n), \varphi \in \mathcal{C}_{c}^{\infty}\left(\mathbb{R}^{d}\right), \widehat{\varphi} \geq 0$ and $\|\widehat{\varphi}\|_{1}=1$.

It is easy to see that $\lim _{n} \widehat{\varphi_{n}} * \mathbf{m}(x)=\mathbf{m}(x)$ for every Lebesgue point $x$ of $\mathbf{m}$. In particular, any continuous and bounded function is normalized.

In order to extend Theorem 3.2 to the class of normalized multipliers, we shall need some previous lemmas. The following one is a direct consequence of the proof of [15. Lemma 2.6] for $G=\mathbb{R}^{d}$.

Lemma 3.4. Let $J \in \mathbb{N}$ and let $\left\{\mathbf{m}_{j}\right\}_{j=1}^{J}$ be a family of $L^{\infty}\left(\mathbb{R}^{d}\right)$ functions. For $f \in \mathcal{S}\left(\mathbb{R}^{d}\right), j=1, \ldots, J$ and $x \in \mathbb{R}^{d}$, let

$$
F_{j, x}(\xi)=S_{\mathbf{m}_{j}}\left(e^{-2 \pi i \xi \cdot} f\right)(x), \quad \xi \in \mathbb{R}^{d} .
$$

Let $\mathcal{K}$ be a compact set. Then, for each $k \in \mathbb{N} \backslash\{0\}$, there exists a finite family $\left\{V_{l}^{k}\right\}_{l=1}^{I_{k}}$ of pairwise disjoint measurable sets in $\mathbb{R}^{d}$ such that

(1) $\mathcal{K} \subset \biguplus_{l=1}^{I_{k}} V_{l}^{k}$

(2) if $l=1, \ldots, I_{k}$ and $\xi, \zeta \in V_{l}^{k}$, then

$$
\left|F_{j, x}(\xi)-F_{j, x}(\zeta)\right| \leq 1 / k,
$$

uniformly on $j \in\{1, \ldots, J\}$ and $x \in \mathbb{R}$.

Another key ingredient is the following version of Marcinkiewicz-Zygmund's inequality, whose proof is analogous to that given in [10, Theorem V.2.9] for $p=q=1$ for linear operators.

Theorem 3.5. Let $\left\{T_{j}\right\}_{j}$ be a countable family of linear operators such that

$$
\left\|\sup _{j}\left|T_{j} f\right|\right\|_{L^{1, \infty}\left(\mathbb{R}^{d}, w\right)} \leq\left\|\left\{T_{j}\right\}_{j}\right\|\|f\|_{L^{1}\left(\mathbb{R}^{d}, w\right)} .
$$

Then

$$
\left\|\sup _{j}\left(\sum_{l}\left|T_{j} f_{l}\right|^{2}\right)^{1 / 2}\right\|\left\|_{L^{1, \infty}\left(\mathbb{R}^{d}, w\right)} \leq \mathfrak{c}_{1}\right\|\left\{T_{j}\right\}_{j}\|\|\left(\sum_{l}\left|f_{l}\right|^{2}\right)^{1 / 2} \|_{L^{1}\left(\mathbb{R}^{d}, w\right)},
$$

where

$$
\mathfrak{c}_{1}:=\inf _{0<r<1} \frac{\sqrt{\pi}}{2\left((1-r) \Gamma\left(1+\frac{r}{2}\right)\right)^{1 / r}} .
$$


For $p>1$, the next lemma is an immediate consequence of Minkowskii's inequality, as $L^{p, \infty}$ is normable, but for $p=1$ the convexity of the space $L^{1, \infty}$ fails. Similar results in the unweighted setting are given by [3, Lemma 2.1] and [4, Theorem 1.2].

Lemma 3.6. Let $\varphi \in L^{1}\left(\mathbb{R}^{d}\right)$ and $\left\{\mathbf{m}_{j}\right\}_{j} \in M_{p, w}^{(\mathfrak{w})}\left(\mathbb{R}^{d}\right)$. Then $\left\{\varphi * \mathbf{m}_{j}\right\}_{j} \in M_{p, w}^{(\mathfrak{w})}\left(\mathbb{R}^{d}\right)$ and

$$
\left\|\left\{\varphi * \mathbf{m}_{j}\right\}_{j}\right\|_{M_{p, w}^{(\mathfrak{w})}\left(\mathbb{R}^{d}\right)} \leq \mathfrak{c}_{p}\|\varphi\|_{L^{1}\left(\mathbb{R}^{d}\right)}\left\|\left\{\mathbf{m}_{j}\right\}_{j}\right\|_{M_{p, w}^{(\mathfrak{w})}\left(\mathbb{R}^{d}\right)},
$$

where $\mathfrak{c}_{p}=p^{\prime}$ if $p>1$ and $\mathfrak{c}_{1}$ is the constant given in (3.3.).

Proof. We shall only prove the case $p=1$. Without loss of generality, we can assume that $\left\{\mathbf{m}_{j}\right\}_{j}$ is a finite family of multipliers of cardinality, say $J \in \mathbb{N}$. For $g \in \mathcal{C}_{c}^{\infty}\left(\mathbb{R}^{d}\right)$,

$$
\int\left(\varphi * \mathbf{m}_{j}\right)(\xi) \widehat{g}(\xi) e^{2 \pi i \xi x} d \xi=\int \varphi(y) e^{2 \pi i x y} S_{\mathbf{m}_{j}}\left(e^{-2 \pi i y \cdot} g\right)(x) d y .
$$

Hence,

$$
\left|S_{\varphi * \mathbf{m}_{j}} g(x)\right| \leq \int|\varphi(y)|\left|S_{\mathbf{m}_{j}}\left(e^{-2 \pi i y \cdot} g\right)(x)\right| d y
$$

and thus

$$
\sup _{1 \leq j \leq J}\left|S_{\varphi * \mathbf{m}_{j}} g(x)\right| \leq \int|\varphi(y)| \sup _{1 \leq j \leq J}\left|S_{\mathbf{m}_{j}}\left(e^{-2 \pi i y \cdot} g\right)(x)\right| d y .
$$

Let us first assume that $\varphi \in L^{1}\left(\mathbb{R}^{d}\right)$ is supported on a compact set $\mathcal{K}$. For each $k \geq 1$ let $\left\{V_{l}^{k}\right\}_{l=1}^{I_{k}}$ be the family of pairwise disjoint sets given by Lemma 3.4. and for each $l$, select $y_{l}^{k} \in V_{l}^{k}$. Then, for every $y \in \mathcal{K}$ and any $k \geq 1$, there exists a unique $l \in\left\{1, \ldots, I_{k}\right\}$ such that $y \in V_{l}^{k}$, and hence

$$
\left|S_{\mathbf{m}_{j}}\left(e^{-2 \pi i y \cdot} g\right)(x)-S_{\mathbf{m}_{j}}\left(e^{-2 \pi i y_{l}^{k} \cdot} g\right)(x)\right| \leq \frac{1}{k},
$$

uniformly on $j=1, \ldots, J$ and $x \in \mathbb{R}^{d}$. It follows that for every $x \in \mathbb{R}^{d}$, any $j \in\{1, \ldots, J\}$ and all $y \in \mathcal{K}$,

$$
\lim _{k} \sum_{l=1}^{I_{k}} S_{\mathbf{m}_{j}}\left(e^{-2 \pi i y_{l}^{k}} \cdot g\right)(x) \chi_{V_{l}^{k}}(y)=S_{\mathbf{m}_{j}}\left(e^{-2 \pi i y \cdot g}\right)(x) .
$$

Then, by Fatou's Lemma on (3.5),

$$
\sup _{1 \leq j \leq J}\left|S_{\varphi * \mathbf{m}_{j}} g(x)\right| \leq \liminf _{k} \sup _{1 \leq j \leq J}\left(\sum_{l=1}^{I_{k}}\left|S_{\mathbf{m}_{j}}\left(e^{-2 \pi i y_{l}^{k}} \cdot g\right)(x)\right| \lambda_{l}^{k}\right),
$$

where $\lambda_{l}^{k}=\int_{V_{l}^{k}}|\varphi(y)| d y$. Observe that the term inside brackets is less than or equal to

$$
\|\varphi\|_{L^{1}\left(\mathbb{R}^{d}\right)}^{1 / 2}\left(\sum_{l=1}^{I_{k}}\left|S_{\mathbf{m}_{j}}\left(\sqrt{\lambda_{l}^{k}} e^{-2 \pi i y_{l}^{k} \cdot g}\right)(x)\right|^{2}\right)^{1 / 2},
$$


where we have used that $\sum_{l=1}^{I_{k}} \lambda_{l}^{k}=\int_{\biguplus_{l=1}^{k} V_{l}^{k}}|\varphi(y)| d y=\|\varphi\|_{L^{1}\left(\mathbb{R}^{d}\right)}$. Then,

$$
\begin{aligned}
& \left\|\sup _{1 \leq j \leq J}\left|S_{\varphi * \mathbf{m}_{j}} g\right|\right\|_{L^{1, \infty}\left(\mathbb{R}^{d}, w\right)} \\
& \quad \leq\|\varphi\|_{L^{1}\left(\mathbb{R}^{d}\right)}^{1 / 2} \liminf _{k}\left\|\sup _{1 \leq j \leq J}\left(\sum_{l=1}^{I_{k}}\left|S_{\mathbf{m}_{j}}\left(\sqrt{\lambda_{l}^{k}} e^{-2 \pi i y_{l}^{k} \cdot g}\right)(x)\right|^{2}\right)^{1 / 2}\right\|_{L^{1, \infty}\left(\mathbb{R}^{d}, w\right)} .
\end{aligned}
$$

Applying Theorem 3.5 with the family of operators $\left\{S_{\mathbf{m}_{j}}\right\}_{j}$ to the functions $f_{l}=$ $\sqrt{\lambda_{l}^{k}} e^{-2 \pi i y_{l}^{k}} \cdot g$, we obtain that

$$
\begin{gathered}
\left\|\sup _{1 \leq j \leq J}\left(\sum_{l=1}^{I_{k}}\left|S_{\mathbf{m}_{j}}\left(\sqrt{\lambda_{l}^{k}} e^{-2 \pi i y_{l}^{k} \cdot g}\right)\right|^{2}\right)^{1 / 2}\right\|_{L^{1, \infty}\left(\mathbb{R}^{d}, w\right)} \\
\leq \mathfrak{c}_{1}\left\|\left\{\mathbf{m}_{j}\right\}_{j}\right\|_{M_{1, w}^{(\mathfrak{r})}\left(\mathbb{R}^{d}\right)} \|\left(\sum_{l=1}^{I_{k}} \mid \sqrt{\lambda_{l}^{k}} e^{\left.-\left.2 \pi i y_{l}^{k} \cdot g\right|^{2}\right)^{1 / 2} \|} \|_{L^{1}\left(\mathbb{R}^{d}, w\right)}\right. \\
=\mathfrak{c}_{1}\left\|\left\{\mathbf{m}_{j}\right\}_{j}\right\|_{M_{1, w}^{(\mathfrak{w})}\left(\mathbb{R}^{d}\right)}\|\varphi\|_{L^{1}\left(\mathbb{R}^{d}\right)}^{1 / 2}\|g\|_{L^{1}\left(\mathbb{R}^{d}, w\right)} .
\end{gathered}
$$

Therefore,

$$
\left\|\sup _{1 \leq j \leq J}\left|S_{\varphi * \mathbf{m}_{j}} g\right|\right\|_{L^{1, \infty}\left(\mathbb{R}^{d}, w\right)} \leq \mathfrak{c}_{1}\|\varphi\|_{L^{1}\left(\mathbb{R}^{d}\right)}\left\|\left\{\mathbf{m}_{j}\right\}_{j}\right\|_{M_{1, w}^{(\mathfrak{w})}\left(\mathbb{R}^{d}\right)}\|g\|_{L^{1}\left(\mathbb{R}^{d}, w\right)} .
$$

In the case that $\varphi$ is not compactly supported, considering $\varphi_{n}=\varphi \chi_{B(0, n)}$, we can write

$$
\sup _{1 \leq j \leq J}\left|S_{\varphi * \mathbf{m}_{j}} g(x)\right| \leq \lim _{n} \int\left|\varphi_{n}(y)\right| \sup _{1 \leq j \leq J}\left|S_{\mathbf{m}_{j}}\left(e^{-2 \pi i y \cdot g}\right)(x)\right| d y,
$$

and using the previous argument we obtain that

$$
\left\|\sup _{1 \leq j \leq J}\left|S_{\varphi * \mathbf{m}_{j}} g\right|\right\|_{L^{1, \infty}\left(\mathbb{R}^{d}, w\right)} \leq \mathfrak{c}_{1} \liminf _{n}\left\|\varphi_{n}\right\|_{L^{1}\left(\mathbb{R}^{d}\right)}\left\|\left\{\mathbf{m}_{j}\right\}_{j}\right\|_{M_{1, w}^{(\mathfrak{w})}\left(\mathbb{R}^{d}\right)}\|g\|_{L^{1}\left(\mathbb{R}^{d}, w\right)},
$$

from where it follows that (3.6) holds for any $\varphi \in L^{1}\left(\mathbb{R}^{d}\right)$. The result now follows by the density of $\mathcal{C}_{c}^{\infty}\left(\mathbb{R}^{d}\right)$ in $L^{p}\left(\mathbb{R}^{d}, w\right)$.

Lemma 3.7. Let $w \in \mathcal{C}\left(\mathbb{T}^{d}\right)$ such that $\inf _{x \in \mathbb{T}^{d}} w(x)>0$. Consider $h \in \mathcal{C}_{c}^{\infty}\left(\mathbb{R}^{d}\right)$ satisfying $0 \leq h \leq 1$ and $\int_{\mathbb{R}^{d}} h=1$ and define $h_{n}(x)=n^{d} h(n x)$. Then,

(1) There exists $n_{0}=n_{0}(w) \in \mathbb{N}$ such that, for any $p \in[1, \infty)$,

$$
\sup _{n \geq n_{0}}\left\|\widehat{h_{n}}\right\|_{M_{p, w}\left(\mathbb{R}^{d}\right)} \leq 2^{1 / p} .
$$

(2) $\sup _{n}\left\|\widehat{h_{n}}\right\|_{L^{\infty}\left(\mathbb{R}^{d}\right)} \leq 1$.

(3) For every $\xi \in \mathbb{R}^{d}, \lim _{n} \widehat{h_{n}}(\xi)=1$.

Proof. Since $\left\|h_{n}\right\|_{L^{1}}=1$, it follows that $\left\|\widehat{h_{n}}\right\|_{\infty} \leq 1$. On the other hand, for every $\xi \in \mathbb{R}^{d}$ and for every $\epsilon>0$ there exists $n_{0}$ such that for all $|x|<\frac{1}{n_{0}},\left|1-e^{2 \pi i x \xi}\right|<\epsilon$. 
Hence, for every $n \geq n_{0}$,

$$
\left|1-\widehat{h_{n}}(\xi)\right| \leq \int h_{n}(x)\left|1-e^{2 \pi i x \xi}\right| d x \leq \epsilon .
$$

Then, it follows that $\widehat{h_{n}} \rightarrow 1$ pointwise. It remains to show that $\left\|\widehat{h_{n}}\right\|_{M_{p, w}\left(\mathbb{R}^{d}\right)}$ are uniformly bounded on $n$.

Observe that $\|f\|_{L^{\infty}(w)}=\|f\|_{L^{\infty}}$ and hence, for any $n \geq 1$,

$$
\left\|h_{n} * f\right\|_{L^{\infty}\left(\mathbb{R}^{d}, w\right)} \leq\|f\|_{L^{\infty}\left(\mathbb{R}^{d}, w\right)} .
$$

Let $\delta=\inf _{x \in \mathbb{T}^{d}} w(x)>0$. Since $w \in \mathcal{C}\left(\mathbb{T}^{d}\right)$, there exists $n_{0}=n_{0}(\delta)$ such that, for any $n \geq n_{0}$, for any $x$ and any $y \in \operatorname{supp} h_{n}$,

$$
|w(x)-w(x-y)| \leq \delta,
$$

which implies that, for any $x \in \mathbb{T}^{d}$,

$$
h_{n} * w(x) \leq \delta+w(x) \leq 2 w(x) .
$$

Then, for $n \geq n_{0}$,

$$
\left\|h_{n} * f\right\|_{L^{1}\left(\mathbb{R}^{d}, w\right)} \leq \int_{\mathbb{R}^{d}}|f(y)| h_{n} * w(y) d y \leq 2\|f\|_{L^{1}\left(\mathbb{R}^{d}, w\right)} .
$$

In other words, we have seen that for $n \geq n_{0}$ the linear operator defined by $T_{n} f=$ $h_{n} * f$ is uniformly bounded on $L^{1}\left(\mathbb{R}^{d}, w\right)$ and $L^{\infty}\left(\mathbb{R}^{d}, w\right)$ with norm respectively bounded by 2 and 1 . Riesz-Thorin's Theorem implies the result.

Lemma 3.8. Let $w$ be 1-periodic. If $g \in \mathcal{C}_{c}\left(\mathbb{R}^{d}\right)$ is nonnegative, $\int_{\mathbb{R}^{d}} g=1$ and $\operatorname{supp} g \subset[-1 / 2,1 / 2]^{d}$, then $\inf _{x \in \mathbb{R}^{d}} g * w(x)>0$.

Proof. Clearly $g * w \in \mathcal{C}\left(\mathbb{T}^{d}\right)$, and hence there exists $x_{0} \in[-1 / 2,1 / 2]^{d}$ such that $\inf _{x \in \mathbb{R}^{d}} g * w(x)=\min _{|x|_{\infty} \leq 1 / 2} g * w(x)=g * w\left(x_{0}\right)$. Since $g \in \mathcal{C}_{c}\left(\mathbb{R}^{d}\right)$, there exists a set of positive Lebesgue measure $Q$ where $g(y)>0$ for all $y \in Q$. Thus if $0=g * w\left(x_{0}\right)=\int g(y) w\left(x_{0}-y\right) d y$, then $g(y) w\left(x_{0}-y\right)=0$ a.e. $y \in Q$, which implies that $w(z)=0$ a.e. $z \in x_{0}-Q$, but this contradicts the fact that the set $\{x: w(x)=0\}$ is null.

Lemma 3.9. Let $T$ be any bounded operator from $L^{p}\left(\mathbb{R}^{d}, w\right)$ to $L^{p, \infty}\left(\mathbb{R}^{d}, w\right)$ that conmutes with translations. Then, for any nonnegative function $g \in \mathcal{C}_{c}\left(\mathbb{R}^{d}\right), T$ is bounded from $L^{p}\left(\mathbb{R}^{d}, g * w\right)$ to $L^{p, \infty}\left(\mathbb{R}^{d}, g * w\right)$ and

$$
\|T\|_{\mathcal{B}\left(L^{p}\left(\mathbb{R}^{d}, g * w\right), L^{p, \infty}\left(\mathbb{R}^{d}, g * w\right)\right)} \leq c_{p}\|T\|_{\mathcal{B}\left(L^{p}\left(\mathbb{R}^{d}, w\right), L^{p, \infty}\left(\mathbb{R}^{d}, w\right)\right)},
$$

where $c_{p}=\inf _{q<p}\left(\frac{p}{p-q}\right)^{\frac{1}{q}}$.

Proof. Let $E$ be any measurable set in $\mathbb{R}^{d}$ such that $0<g * w(E)<+\infty$. Then, for any $q<p$,

$$
\begin{aligned}
\left\|T f \chi_{E}\right\|_{L^{q}\left(\mathbb{R}^{d}, g * w\right)}^{q} & =\int_{E}|T f(x)|^{q} g * w(x) d x=\int g(y) \int_{E}|T f(x)|^{q} w(x-y) d x d y \\
& =\int g(y) \int_{E-y}\left|T f_{y}(x)\right|^{q} w(x) d x d y,
\end{aligned}
$$


with $f_{y}(z)=f(z+y)$. Thus, by the boundedness hypothesis, Kolmogorov's condition and Hölder's inequality,

$$
\begin{aligned}
\left\|T f \chi_{E}\right\|_{L^{q}\left(\mathbb{R}^{d}, g * w\right)}^{q} & \leq\|T\|^{q} \int g(y) w(E-y)^{1-\frac{q}{p}}\left(\int\left|f_{y}(x)\right|^{p} w(x) d x\right)^{q / p} d y \\
& \leq c_{p, q}^{q}\|T\|^{q}(g * w(E))^{1-\frac{q}{p}}\|f\|_{L^{p}\left(\mathbb{R}^{d}, g * w\right)}^{q},
\end{aligned}
$$

where $c_{p, q}^{q}=p /(p-q)$. Then, the result follows by Kolmogorov's condition and by taking the infimum for $q<p$.

Theorem 3.10. Let $w$ be a 1-periodic weight on $\mathbb{R}^{d}$. Suppose that $\left\{\mathbf{m}_{j}\right\}_{j}$ are normalized functions and $\left\{\mathbf{m}_{j}\right\}_{j} \in M_{p, w}^{(\mathfrak{w})}\left(\mathbb{R}^{d}\right)$. Then $\left\{\left.\mathbf{m}_{j}\right|_{\mathbb{Z}^{d}}\right\}_{j} \in M_{p, w}^{(\mathfrak{w})}\left(\mathbb{T}^{d}\right)$ and

$$
\|\left\{\mathbf{m}_{j}{\left.\mid \mathbb{Z}^{d}\right\}_{j}}_{\|_{M_{p, w}^{(\mathfrak{w})}\left(\mathbb{T}^{d}\right)}} \leq c_{p}\left\|\left\{\mathbf{m}_{j}\right\}_{j}\right\|_{M_{p, w}^{(\mathfrak{w})}\left(\mathbb{R}^{d}\right)},\right.
$$

where $c_{p}$ depends only on $p$.

Proof. Let $\left\{g_{l}\right\}_{l}$ be a family of nonnegative functions in $\mathcal{C}_{c}^{\infty}\left(\mathbb{R}^{d}\right)$, supported in $[-1 / 2,1 / 2]^{d}$ such that it is an approximation of the identity in $L^{1}\left(\mathbb{T}^{d}\right)$. We can also assume that $\lim _{l} g_{l} * w(x)=w(x)$ a.e. $x \in[-1 / 2,1 / 2]^{d}$.

For a fixed $l \in \mathbb{N}$, by Lemma 3.9.

$$
\left\|\left\{\mathbf{m}_{j}\right\}_{j}\right\|_{M_{p, g_{l} * w}^{(\mathfrak{w})}\left(\mathbb{R}^{d}\right)} \leq c_{p}\left\|\left\{\mathbf{m}_{j}\right\}_{j}\right\|_{M_{p, w}^{(\mathfrak{w})}\left(\mathbb{R}^{d}\right)} .
$$

By Lemma 3.8 it follows that for any $h \in \mathcal{C}_{c}^{\infty}\left(\mathbb{R}^{d}\right)$ such that $0 \leq h \leq 1$ and that $\int_{\mathbb{R}^{d}} h=1$, there exists an $n_{l}$ such that, for any $n \geq n_{l}$, the conclusions of Lemma 3.7 hold for the periodic weight $g_{l} * w$.

Consider, for $j, n \in \mathbb{N}$,

$$
\mathbf{m}_{j, n}(\xi)=\widehat{K_{j, n}}(\xi)=\left(\widehat{\varphi_{n}} * \mathbf{m}_{j}\right)(\xi) \widehat{h_{n}}(\xi),
$$

where $\varphi_{n}$ are the functions given by the normalized condition. First observe that $\widehat{K_{j, n}} \in \mathcal{S}\left(\mathbb{R}^{d}\right)$ and hence $K_{j, n} \in \mathcal{S}\left(\mathbb{R}^{d}\right)$. Moreover, since

$$
K_{j, n}(x)=\left(\varphi_{n} \mathbf{m}_{j}^{\vee}\right)\left(h_{n}(x-\cdot)\right)=\mathbf{m}_{j}^{\vee}\left(\varphi_{n}(\cdot) h_{n}(x-\cdot)\right),
$$

and $\varphi_{n}, h_{n}$ are compactly supported, it follows that $K_{j, n} \in \mathcal{C}_{c}^{\infty}\left(\mathbb{R}^{d}\right)$. On the other hand, since $\mathbf{m}_{j}$ is normalized and $\widehat{h_{n}} \rightarrow 1$, it holds that for every $\xi \in \mathbb{R}^{d}$,

$$
\lim _{n} \widehat{K_{j, n}}(\xi)=\mathbf{m}_{j}(\xi) \text {. }
$$

Since $\left\|\widehat{h_{n}}\right\|_{L^{\infty}\left(\mathbb{R}^{d}\right)} \leq 1$ and $\left\|\widehat{\varphi_{n}}\right\|_{L^{1}\left(\mathbb{R}^{d}\right)} \leq 1$, then $\left\|\mathbf{m}_{j, n}\right\|_{L^{\infty}\left(\mathbb{R}^{d}\right)} \leq\left\|\mathbf{m}_{j}\right\|_{L^{\infty}\left(\mathbb{R}^{d}\right)}$.

Let us fix $J \in \mathbb{N}$. Since for any $f \in \mathcal{C}_{c}^{\infty}(\mathbb{R})$

$$
K_{j, n} * f=T_{\widehat{\varphi_{n}} * \mathbf{m}_{j}}\left(h_{n} * f\right),
$$

it follows that for every $n \geq n_{l}$,

$$
\begin{aligned}
\left\|\sup _{1 \leq j \leq J}\left|K_{j, n} * f\right|\right\|_{L^{p, \infty}\left(\mathbb{R}^{d}, g_{l} * w\right)} & \leq\left\|\left\{\widehat{\varphi_{n}} * \mathbf{m}_{j}\right\}_{j}\right\|_{M_{p, g_{l} * w}^{(\mathfrak{w})}\left(\mathbb{R}^{d}\right)}\left\|h_{n} * f\right\|_{L^{p}\left(\mathbb{R}^{d}, g_{l} * w\right)} \\
& \leq c_{p} 2^{\frac{1}{p}}\left\|\left\{\mathbf{m}_{j}\right\}_{j}\right\|_{M_{p, w}^{(\mathfrak{w})}\left(\mathbb{R}^{d}\right)}\|f\|_{L^{p}\left(\mathbb{R}^{d}, g_{l} * w\right)},
\end{aligned}
$$


where we have used that, by Lemma 3.6.

$$
\left\|\left\{\widehat{\varphi_{n}} * \mathbf{m}_{j}\right\}_{j}\right\|_{M_{p, g_{l} * w}^{(\mathfrak{w})}\left(\mathbb{R}^{d}\right)} \leq c_{p}\left\|\left\{\mathbf{m}_{j}\right\}_{j}\right\|_{M_{p, g_{l} * w}^{(\mathfrak{w})}\left(\mathbb{R}^{d}\right)} \leq c_{p}^{2}\left\|\left\{\mathbf{m}_{j}\right\}_{j}\right\|_{M_{p, w}^{(\mathfrak{w})}\left(\mathbb{R}^{d}\right)} .
$$

We can now apply Theorem 3.2 to deduce that for any $n \geq n_{l}$,

$$
\left\|\left\{\left.\mathbf{m}_{j, n}\right|_{\mathbb{Z}^{d}}\right\}_{j=1}^{J}\right\|_{M_{p, g_{l} * w}^{(\mathfrak{w})}\left(\mathbb{T}^{d}\right)} \leq 2^{\frac{1}{p}} c_{p}^{2}\left\|\left\{\mathbf{m}_{j}\right\}_{j}\right\|_{M_{p, w}^{(\mathfrak{w})}\left(\mathbb{R}^{d}\right)} .
$$

Since, for any $f \in P\left(\mathbb{T}^{d}\right)$,

$$
\lim _{n} S_{\mathbf{m}_{j, n}} f(s)=\lim _{n} \sum_{k \in \mathbb{Z}^{d}} \mathbf{m}_{j, n}(k) \widehat{f}(k) e^{2 \pi i k s}=\sum_{k \in \mathbb{Z}^{d}} \mathbf{m}_{j}(k) \widehat{f}(k) e^{2 \pi i k s}=S_{\mathbf{m}_{j}} f(s),
$$

by Fatou's Lemma, the following inequality holds:

$$
\begin{array}{r}
\left\|\sup _{1 \leq j \leq J}\left|S_{\mathbf{m}_{j}} f\right|\right\|_{L^{p, \infty}\left(\mathbb{T}^{d}, g_{l} * w\right)} \\
\leq \liminf _{n}\left\|\sup _{1 \leq j \leq J}\left|S_{\mathbf{m}_{j, n}} f\right|\right\|_{L^{p, \infty}\left(\mathbb{T}^{d}, g_{l} * w\right)} \\
\leq 2^{\frac{1}{p}} c_{p}^{2}\left\|\left\{\mathbf{m}_{j}\right\}_{j}\right\|_{M_{p, w}^{(\mathfrak{w})}\left(\mathbb{R}^{d}\right)}\|f\|_{L^{p}\left(\mathbb{T}^{d}, g_{l} * w\right)},
\end{array}
$$

which implies

$$
\left\|\sup _{1 \leq j}\left|S_{\mathbf{m}_{j}} f\right|\right\|_{L^{p, \infty}\left(\mathbb{T}^{d}, w\right)} \leq 2^{\frac{1}{p}} c_{p}^{2}\left\|\left\{\mathbf{m}_{j}\right\}_{j}\right\|_{M_{p, w}^{(\mathfrak{w})}\left(\mathbb{R}^{d}\right)} \liminf _{l \rightarrow \infty}\|f\|_{L^{p}\left(\mathbb{T}^{d}, g_{l} * w\right)} .
$$

Observe that

$$
\|f\|_{L^{p}\left(\mathbb{T}^{d}, g_{l} * w\right)} \leq\|f\|_{L^{\infty}\left(\mathbb{T}^{d}\right)}\left\|g_{l} * w-w\right\|_{L^{1}\left(\mathbb{T}^{d}\right)}+\|f\|_{L^{p}\left(\mathbb{T}^{d}, w\right)},
$$

and since $\lim _{l}\left\|g_{l} * w-w\right\|_{L^{1}\left(\mathbb{T}^{d}\right)}=0$, it follows that

$$
\left\|\sup _{1 \leq j}\left|S_{\mathbf{m}_{j}} f\right|\right\|_{L^{p, \infty\left(\mathbb{T}^{d}, w\right)}} \leq 2^{\frac{1}{p}} c_{p}^{2}\|f\|_{L^{p}\left(\mathbb{T}^{d}, w\right)} .
$$

The result follows by the density of $P\left(\mathbb{T}^{d}\right)$ in $L^{p}\left(\mathbb{T}^{d}, w\right)$.

With minor modifications in the proofs, the analogous result for operators of strong type can be proved. In the particular case of a single multiplier, we recover K. Andersen and P. Mohanty's [1, Theorem 1.1].

Theorem 3.11. Let $w$ be 1-periodic. Suppose that $\left\{\mathbf{m}_{j}\right\}_{j} \subset M_{p, w}\left(\mathbb{R}^{d}\right)$ and that they are normalized functions. Then $\left\{\left.\mathbf{m}_{j}\right|_{\mathbb{Z}^{d}}\right\}_{j} \subset M_{p, w}\left(\mathbb{T}^{d}\right)$ and

$$
\left\|\left\{\left.\mathbf{m}_{j}\right|_{\mathbb{Z}^{d}}\right\}_{j}\right\|_{M_{p, w}\left(\mathbb{T}^{d}\right)} \leq 2^{1 / p}\left\|\left\{\mathbf{m}_{j}\right\}_{j}\right\|_{M_{p, w}\left(\mathbb{R}^{d}\right)} .
$$

3.2. An improvement for nonperiodic weights. A similar approach to that in the previous section allows us to obtain a more general version of Theorem 3.10 (and also of Theorem 3.11) for a class of nonnecessarily periodic weights which includes those in $A_{p}\left(\mathbb{T}^{d}\right)$.

Definition 3.12. We say that a weight $v \in W\left(\mathbb{R}^{d}\right)$ if it satisfies the following conditions:

i) For every $x \in \mathbb{R}^{d}, \theta \in[0,1)^{d}$,

$$
\frac{1}{\zeta} \leq \frac{v(x)}{v(x+\theta)} \leq \zeta
$$


ii)

$$
\lim _{s \rightarrow \infty} \frac{v\left(Q_{r+s}\right)}{v\left(Q_{s}\right)}=1
$$

Theorem 3.13. Let $u$ be a periodic weight in $\mathbb{R}^{d}$, let $v \in W$ and set $w=u v$. Assume that $\left\{\mathbf{m}_{j}\right\}_{j} \in M_{p, w}^{(\mathfrak{w})}\left(\mathbb{R}^{d}\right)$ (respectively $M_{p, w}\left(\mathbb{R}^{d}\right)$ ) and that they are normalized functions in $\mathbb{R}^{d}$. Then $\left\{\left.\mathbf{m}_{j}\right|_{\mathbb{Z}^{d}}\right\}_{j} \in M_{p, w}^{(\mathfrak{w})}\left(\mathbb{T}^{d}\right)$ (respectively $M_{p, w}\left(\mathbb{T}^{d}\right)$ ) and

$$
\left\|\left\{\left.\mathbf{m}_{j}\right|_{\mathbb{Z}^{d}}\right\}_{j}\right\|_{M_{p, u}^{(\mathfrak{w})}\left(\mathbb{T}^{d}\right)} \leq \mathfrak{c}_{p, w} c_{p, v}\left\|\left\{\mathbf{m}_{j}\right\}_{j}\right\|_{M_{p, u}^{(\mathfrak{w})}\left(\mathbb{R}^{d}\right)}
$$

(respectively replacing $M_{p, u}^{(\mathfrak{w})}$ with $M_{p, u}$ in the previous inequality), where $\mathfrak{c}_{p, w}$ and $c_{p, v}$ depend only on $p$.

Proof. We shall prove the weak case. The proof for the strong case is similar and we leave the details to the reader. Assume first that $\left\{\mathbf{m}_{j}\right\}_{j}$ is a finite sequence. The argument is similar to that for Theorem 3.2 , and we shall sketch the major changes to be done in the proof.

Let $\mathfrak{N}=\left\|\left\{\mathbf{m}_{j}\right\}_{j}\right\|_{M_{p, w}^{(\mathfrak{w})}\left(\mathbb{R}^{d}\right)}$. Since $v \in W$,

$$
\frac{1}{\zeta} w(x+\theta) \leq u(x+\theta) v(x) \leq \zeta w(x+\theta) .
$$

By (3.2), for every $f \in P(\mathbb{T})$ and every measurable set $E \subset \mathbb{T}$,

$$
\begin{aligned}
& \left\|\sup _{1 \leq j \leq N}\left|T_{K_{j}} f\right| \chi_{E}\right\|_{L^{q}\left(\mathbb{T}^{d}, u\right)}^{q} \\
& =\frac{1}{v\left(Q_{s}\right)} \int_{Q_{s}} \int_{\mathbb{T}^{d}} \sup _{1 \leq j \leq N}\left|R_{x} T_{K_{j}} f(\theta)\right|^{q} u(x+\theta) v(x) \chi_{\tilde{E}}(x+\theta) d \theta d x \\
& \leq \frac{\zeta}{v\left(Q_{s}\right)} \int_{\mathbb{T}^{d}}\left\{\int_{E_{\theta} \cap Q_{s}} \sup _{1 \leq j \leq N}\left|B_{K_{j}}\left(R_{(\cdot)} f(\theta) \chi_{Q_{r+s}}\right)(x)\right|^{q} w(x+\theta) d x\right\} d \theta,
\end{aligned}
$$

where $E_{\theta}=\left\{x \in \mathbb{R}^{d}: x+\theta \in \tilde{E}\right\}$ and $\tilde{E}$ is the periodic extension of $E$. By (2.1) and (3.1), the term inside curly brackets is bounded by

$$
\left(c_{p, q} \mathfrak{N}\right)^{q}\left\{\int_{Q_{r+s}}\left|R_{x} f(\theta)\right|^{p} w(x+\theta) d t\right\}^{\frac{q}{p}}\left\{\int_{E_{\theta} \cap Q_{s}} w(x+\theta) d t\right\}^{1-\frac{q}{p}} .
$$

Hence, by Hölder's inequality, it follows that

$$
\begin{aligned}
& \left\|\sup _{1 \leq j \leq N}\left|T_{K_{j}} f\right| \chi_{E}\right\|_{L^{q}\left(\mathbb{T}^{d}, u\right)}^{q} \\
& \leq \frac{c_{p, q}^{q} \mathfrak{N}^{q} \zeta}{v\left(Q_{s}\right)}\left[\int_{\mathbb{T}^{d}} \int_{Q_{r+s}}\left|R_{x} f(\theta)\right|^{p} w(x+\theta) d t d \theta\right]^{\frac{q}{p}}\left[\int_{\mathbb{T}^{d}} \int_{Q_{s} \cap E_{\theta}} w(x+\theta) d x d \theta\right]^{1-\frac{q}{p}} .
\end{aligned}
$$

By (3.7), the first term is bounded by $\left[\zeta v\left(Q_{r+s}\right)\right]^{\frac{q}{p}}\|f\|_{L^{p}(\mathbb{T}, u)}^{q}$, and the second one by $\left[\zeta v\left(Q_{s}\right) u(E)\right]^{1-\frac{q}{p}}$. Hence,

$$
u(E)^{\frac{q}{p}-1}\left\|\sup _{1 \leq j \leq N}\left|T_{K_{j}} f\right| \chi_{E}\right\|_{L^{q}\left(\mathbb{T}^{d}, u\right)}^{q} \leq c_{p, q}^{q} \mathfrak{N}^{q} \zeta^{2}\left(\frac{v\left(Q_{r+s}\right)}{v\left(Q_{s}\right)}\right)^{\frac{q}{p}}\|f\|_{L^{p}\left(\mathbb{T}^{d}, u\right)}^{q} .
$$


Letting $s \rightarrow \infty$ and using (2.1) we obtain that

$$
\left\|\sup _{1 \leq j \leq N}\left|T_{K_{j}} f\right|\right\|_{L^{p, \infty}\left(\mathbb{T}^{d}, u\right)} \leq c_{p, q} \zeta^{\frac{2}{q}} \mathfrak{N}\|f\|_{L^{p}\left(\mathbb{T}^{d}, u\right)} .
$$

Considering $c_{p, v}=\inf _{q<p} \zeta^{2 / q} c_{p, q}$, the result easily follows by Fatou's Lemma and the density of $P\left(\mathbb{T}^{d}\right)$ in $L^{p}\left(\mathbb{T}^{d}, u\right)$.

\section{Restriction of Fourier multipliers to LOWER Dimension}

Restriction of Fourier multipliers of strong type to a lower dimensional space was studied in [7, Corollary 4.13]. Here we shall give a weak counterpart to that result.

We have to mention here that in this section we work with $A_{p}\left(\mathbb{R}^{d}\right)$ weights mainly because, under this condition, we can prove the analogue to Lemma 3.7 (see Lemma 4.2 below). Other conditions that we can assume in $w$ in order to have an approximation lemma are, for example, that $w$ is uniformly continuous and $\inf _{x \in \mathbb{R}^{d}} w(x)>0$. In this case the proof is a simple modification of the proof of Lemma 3.7 .

Lemma 4.1. If $w \in \bigcup_{1 \leq p<\infty} A_{p}\left(\mathbb{R}^{d}\right)$, then, for any $s>0, \lim _{s \rightarrow \infty} \frac{w\left(Q_{r+s}\right)}{w\left(Q_{s}\right)}=1$.

Proof. By the $A_{\infty}$-condition [10, Theorem IV.2.9], there exist $\delta, C>0$ such that

$$
0 \leq 1-\frac{w\left(Q_{s}\right)}{w\left(Q_{r+s}\right)}=\frac{w\left(Q_{r+s} \backslash Q_{s}\right)}{w\left(Q_{r+s}\right)} \leq C\left(1-\frac{s^{d}}{(r+s)^{d}}\right)^{\delta},
$$

from where the result easily follows.

Lemma 4.2. If $w \in A_{p}\left(\mathbb{R}^{d}\right)$, there exists $\left\{h_{n}\right\}_{n} \subset \mathcal{C}_{c}^{\infty}\left(\mathbb{R}^{d}\right)$, such that

(1) $\mathfrak{s}_{p, w}:=\sup _{n}\left\|\widehat{h_{n}}\right\|_{M_{p, w}\left(\mathbb{R}^{d}\right)}<\infty$,

(2) $\sup _{n}\left\|\widehat{h_{n}}\right\|_{L^{\infty}\left(\mathbb{R}^{d}\right)} \leq 1$,

(3) for every $\xi \in \mathbb{R}^{d}, \lim _{n} \widehat{h_{n}}(\xi)=1$.

Proof. Properties (2) and (3) are proved as in Lemma 3.7 To prove (1), we first observe that clearly

$$
\sup _{n}\left|h_{n} * f\right|(x) \lesssim M f(x)
$$

and hence the case $p>1$ is trivial.

To prove the case $p=1$, fix $\beta \in \mathbb{N}, \beta>d$. Then,

$$
\left\|h_{n} * f\right\|_{L^{1}(w)} \leq \int|f(y)| \int h_{n}(x-y) w(x) d x d y .
$$

For a fixed $y \in \mathbb{R}^{d}$ and $n>0$, the inner integral can be split into

$$
\int_{|x-y|<n^{-1}}+\sum_{j \geq 0} \int_{2^{j} n^{-1}<|x-y| \leq 2^{j+1} n^{-1}} h(n(x-y)) n^{d} w(x) d x .
$$

The first term can be bounded by

$$
\|h\|_{\infty} n^{d} \int_{|x-y|<n^{-1}} w(x) d x \leq[w]_{A_{1}} 2^{d} w(y),
$$


as the ball $|x-y|<1 / n$ is included in $y+[-1 / n, 1 / n]^{d}$. On the other hand, if $p_{0, \beta}(h)=\sup _{x \in \mathbb{R}^{d}}|h(x)||x|^{\beta}$, each term on the sum can be bounded from above by

$$
p_{0, \beta}(h) n^{d-\beta} \int_{2^{j}<n|x-y| \leq 2^{j+1}}|x-y|^{-\beta} w(x) d x \leq p_{0, \beta}(h) 4^{d 2^{j(d-\beta)}}[w]_{A_{1}} w(y) .
$$

Thus, the sum is bounded from above by $\frac{p_{0, \beta}(h) 4^{d}}{1-2^{d-\beta}}[w]_{A_{1}} w(y)$. Hence

$$
\left\|h_{n} * f\right\|_{L^{1}(w)} \leq[w]_{A_{1}} c_{d, h}\|f\|_{L^{1}(w)},
$$

where $c_{d, h}=2^{d}\left(1+\inf _{\beta>d} p_{0, \beta}(h) \frac{2^{d}}{1-2^{d-\beta}}\right)$.

The following result is the weighted version of [6. Lemma 2] and the weak type maximal counterpart of [7, Proposition 4.10].

Proposition 4.3. Let $w \in A_{p}\left(\mathbb{R}^{d}\right)$ and let $\left\{\mathbf{m}_{j}\right\}_{j} \subset M_{p, w}^{(\mathfrak{w})}\left(\mathbb{R}^{d}\right) \cap L^{\infty}\left(\mathbb{R}^{d}\right)$ that are normalized functions. Then, there exist $\left\{\mathbf{m}_{j, n}\right\}_{j, n} \subset L^{\infty}\left(\mathbb{R}^{d}\right)$ satisfying:

(1) For any $j$ and every $\xi \in \mathbb{R}^{d}$,

$$
\mathbf{m}_{j}(\xi)=\lim _{n} \mathbf{m}_{j, n}(\xi) .
$$

(2) $K_{j, n}=\mathbf{m}_{j, n} \vee \in L^{1}\left(\mathbb{R}^{d}\right)$, and it is compactly supported.

(3) $\sup _{n}\left\|\mathbf{m}_{j, n}\right\|_{L^{\infty}\left(\mathbb{R}^{d}\right)} \leq\left\|\mathbf{m}_{j}\right\|_{L^{\infty}\left(\mathbb{R}^{d}\right)}$.

(4) $\sup _{n}\left\|\left\{\mathbf{m}_{j, n}\right\}_{j}\right\|_{M_{p, w}^{(\mathfrak{w})}\left(\mathbb{R}^{d}\right)} \leq \mathfrak{d}_{p, w}\left\|\left\{\mathbf{m}_{j}\right\}\right\|_{M_{p, w}^{(\mathfrak{w})}\left(\mathbb{R}^{d}\right)}$, where $\mathfrak{d}_{p, w}$ depends only on $p, d$ and the $A_{p}$-constant of $w$.

Proof. Let $\left\{h_{n}\right\}$ be the functions given by Lemma 4.2 and $\varphi_{n}(x)$ as in Definition 3.3. Consider, for $j, n \in \mathbb{N}$,

$$
m_{j, n}(\xi)=\widehat{K_{j, n}}(\xi)=\left(\widehat{\varphi_{n}} * \mathbf{m}_{j}\right)(\xi) \widehat{h_{n}}(\xi),
$$

and proceed as in the proof of Theorem 3.10 .

Theorem 4.4. Let $d=d_{1}+d_{2}, u \in A_{p}\left(\mathbb{R}^{d_{1}}\right), v \in A_{p}\left(\mathbb{R}^{d_{2}}\right)$ and define $w(x, y)=$ $u(x) v(y)$. Suppose that $\left\{\mathbf{m}_{j}\right\}_{j} \in M_{p, w}^{(\mathfrak{w})}\left(\mathbb{R}^{d}\right)$ and are normalized functions. Then, for a fixed $\xi \in \mathbb{R}^{d_{1}},\left\{\mathbf{m}_{j}(\xi, \cdot)\right\}_{j} \in M_{p, w}^{(\mathfrak{w})}\left(\mathbb{R}^{d_{2}}\right)$ and

$$
\sup _{\xi \in \mathbb{R}^{d_{1}}}\left\|\left\{\mathbf{m}_{j}(\xi, \cdot)\right\}_{j}\right\|_{M_{p, u}^{(\mathfrak{w})}\left(\mathbb{R}^{\left.d_{2}\right)}\right.} \leq \mathfrak{c}_{p, w}\left\|\left\{\mathbf{m}_{j}\right\}_{j}\right\|_{\mathbf{m} \in M_{p, u}^{(\mathfrak{w})}\left(\mathbb{R}^{d}\right)},
$$

where $\mathfrak{c}_{p, w}$ depends only on $p, d$ and the $A_{p}$-constant of $w$.

Proof. Since $u \in A_{p}\left(\mathbb{R}^{d_{1}}\right)$ and $v \in A_{p}\left(\mathbb{R}^{d_{2}}\right)$ we have that $w \in A_{p}\left(\mathbb{R}^{d}\right)$ and $[w]_{A_{p}\left(\mathbb{R}^{d}\right)}$ $\leq[v]_{A_{p}\left(\mathbb{R}^{\left.d_{1}\right)}\right.}[u]_{A_{p}\left(\mathbb{R}^{\left.d_{2}\right)}\right)}$. Then, by Proposition 4.3 , we can assume that $\left\{\mathbf{m}_{j}\right\}_{j=1}^{J}$ is a finite family such that $K_{j}=\mathbf{m}_{j}^{\vee} \in L^{1}$ with compact support.

Let $\mathfrak{N}=\left\|\left\{\mathbf{m}_{j}\right\}_{j}\right\|_{M_{p, w}^{(\mathfrak{w})}\left(\mathbb{R}^{d}\right)}$. Since translations and convolution commute, it follows that for every $z \in \mathbb{R}^{d_{2}}$,

$$
\left\|\sup _{1 \leq j \leq J}\left|B_{K_{j}} g\right|\right\|_{L^{p, \infty}\left(\mathbb{R}^{d}, u(\cdot) v(\cdot+z)\right)} \leq \mathfrak{N}\|g\|_{L^{p}\left(\mathbb{R}^{d}, u(\cdot) v(\cdot+z)\right)} .
$$

Fix $\xi \in \mathbb{R}^{d_{1}}$. For any $f \in \mathcal{C}_{c}^{\infty}\left(\mathbb{R}^{d_{2}}\right)$, write

$$
R_{(x, y)} f(z)=e^{2 \pi i x \xi} f(z+y), \quad(x, y) \in \mathbb{R}^{d_{1}} \times \mathbb{R}^{d_{2}} .
$$


Observe that in this way,

$$
\begin{aligned}
\tilde{T}_{K_{j}} f(z) & =\int_{\mathbb{R}^{d}} K_{j}(x, y) R_{-(x, y)} f(z) d x d y \\
& =\int_{\mathbb{R}^{d_{2}}}\left(\int_{\mathbb{R}^{d_{1}}} K_{j}(x, y) e^{-2 \pi i \xi x} d x\right) f(z-y) d y \\
& =\int_{\mathbb{R}^{d_{2}}} \mathbf{m}_{j}(\xi, \eta) \widehat{f}(\eta) e^{-2 \pi i z \eta} d \eta .
\end{aligned}
$$

Fix $q<p$ and fix $E \subset \mathbb{R}^{d_{1}}$ a set of finite measure. For any $z \in \mathbb{R}^{d_{2}}$, let $A_{z}=$ $\left\{(x, y) \in \mathbb{R}^{d}: y+z \in E\right\}$. Let $r>0$ such that $\operatorname{supp} K_{j} \subset(-r, r)^{d}=Q_{r}$ for $j=1, \ldots, J$.

Let $s>0$. For any $(x, y) \in Q_{s}=(-s, s)^{d}$,

$$
\left\|\sup _{1 \leq j \leq J}\left|\tilde{T}_{K_{j}} f\right| \chi_{E}\right\|_{L^{q}\left(\mathbb{R}^{d_{2}}, w\right)}^{q}=\int_{\mathbb{R}^{d_{2}}} \sup _{1 \leq j \leq J}\left|R_{(x, y)} T_{K_{j}} f(z)\right|^{q} v(y+z) \chi_{E}(y+z) d z .
$$

If we consider the weight $\omega=u \otimes 1$ on $\mathbb{R}^{d}$, it follows that

$$
\begin{aligned}
& \left\|\sup _{1 \leq j \leq J}\left|\tilde{T}_{K_{j}} f\right| \chi_{E}\right\|_{L^{q}\left(\mathbb{T}^{d}, w\right)}^{q} \\
& =\frac{1}{\omega\left(Q_{s}\right)} \int_{Q_{s}} \int_{\mathbb{R}^{d_{2}}} \sup _{1 \leq j \leq J}\left|R_{(x, y)} \tilde{T}_{K_{j}} f(z)\right|^{q} u(x) v(y+z) \chi_{E}(y+z) d x d y d z \\
& \leq \frac{1}{\omega\left(Q_{s}\right)} \int_{\mathbb{R}^{d_{2}}}\left\{\int_{A_{z} \cap Q_{s}} \sup _{1 \leq j \leq J}\left|B_{K_{j}}\left(R_{(\cdot)} f(z) \chi_{Q_{r+s}}\right)(x, y)\right|^{q} u(x) v(y+z) d x d y\right\} d z .
\end{aligned}
$$

By Kolmogorov's condition (2.1) and (4.2), the term inside curly brackets is bounded by

$$
\left(c_{p, q} \mathfrak{N}\right)^{q}\left\{\int_{Q_{r+s}}\left|R_{(x, y)} f(z)\right|^{p} u(x) v(y+z) d t\right\}^{\frac{q}{p}}\left\{\int_{A_{z} \cap Q_{s}} u(x) v(y+z) d t\right\}^{1-\frac{q}{p}} .
$$

Then, by Hölder's inequality, it follows that

$$
\begin{aligned}
\left\|\sup _{1 \leq j \leq J}\left|T_{K_{j}} f\right| \chi_{E}\right\|_{L^{q}\left(\mathbb{R}^{\left.d_{2}, v\right)}\right.}^{q} \leq & \frac{c_{p, q}^{q} \mathfrak{N}^{q}}{\omega\left(Q_{s}\right)}\left\{\int_{\mathbb{R}^{d_{2}}} \int_{Q_{s} \cap A_{z}} u(x) v(y+z) d t d z\right\}^{1-\frac{q}{p}} \\
& \times\left\{\int_{\mathbb{R}^{d_{2}}} \int_{Q_{r+s}}\left|R_{(x, y)} f(z)\right|^{p} u(x) v(y+z) d x d y d z\right\}^{\frac{q}{p}} \\
\leq & c_{p, q}^{q} \mathfrak{N}^{q}\left(\frac{\omega\left(Q_{r+s}\right)}{\omega\left(Q_{s}\right)}\right)^{\frac{q}{p}} v(E)^{1-\frac{q}{p}}\|f\|_{L^{p}\left(\mathbb{R}^{d_{2}}, w\right)}^{q} .
\end{aligned}
$$

Since $u \in A_{p}\left(\mathbb{R}^{d_{1}}\right), \omega \in A_{p}\left(\mathbb{R}^{d}\right)$. Then by Lemma 4.1 and Kolmogorov's condition (2.1), it follows that

$$
\left\|\sup _{1 \leq j \leq J}\left|T_{K_{j}} f\right|\right\|_{L^{p, \infty}\left(\mathbb{R}^{d_{2}}, w\right)} \leq c_{p, q} \mathfrak{N}\|f\|_{L^{p}\left(\mathbb{R}^{d_{2}}, w\right)} .
$$

Finally, considering $c_{p}=\inf _{q<p} c_{p, q}$, the result easily follows by Fatou's Lemma and the density of $\mathcal{C}_{c}^{\infty}\left(\mathbb{R}^{d_{2}}\right)$ in $L^{p}\left(\mathbb{R}^{d_{2}}, w\right)$. 


\section{Consequences And applications}

5.1. Hörmander-Mihlin type multipliers. The first application involves multipliers satisfying a Hörmander-Mihlin type condition.

Definition 5.1 (see [14]). Let $\mathbf{m} \in L^{\infty}\left(\mathbb{R}^{d}\right) \cap \mathcal{C}^{d}\left(\mathbb{R}^{d} \backslash\{0\}\right), l \in \mathbb{N}$ and $s \geq 1$. We say $\mathbf{m} \in M(s, l)$ if it satisfies

$$
c_{\mathbf{m}, s, l}=\sup _{\substack{|\alpha| \leq l \\ \alpha=\left(\alpha_{1}, \ldots, \alpha_{d}\right)}} \sup _{r>0}\left(r^{s|\alpha|-d} \int_{r<|x|<2 r}\left|\frac{\partial^{|\alpha|} \mathbf{m}}{\partial x_{1}^{\alpha_{1}} \ldots \partial x_{d}^{\alpha_{d}}}(x)\right|^{s} d x\right)^{1 / s}<\infty .
$$

In 1979, D. Kurtz and R. Wheeden proved the following result.

Theorem 5.2 ([14, Theorem 1]). Let $1<s \leq 2, \frac{d}{s}<l \leq d$ and $\mathbf{m} \in M(s, l)$. If

(1) $d / l<p<\infty$ and $w \in A_{p l / d}\left(\mathbb{R}^{d}\right)$ or

(2) $1<p<(d / l)^{\prime}$ and $w^{-1 /(p-1)} \in A_{p^{\prime} l / d}\left(\mathbb{R}^{d}\right)$,

then $\mathbf{m} \in M_{p, w}\left(\mathbb{R}^{d}\right)$. When $l<d$ it can be taken $p=d / l$ in (1) or $p=(d / l)^{\prime}$ in (2).

Moreover, if $w^{d / l} \in A_{1}\left(\mathbb{R}^{d}\right)$, then $\mathbf{m} \in M_{1, w}^{(\mathfrak{w})}\left(\mathbb{R}^{d}\right)$.

Corollary 5.3. Under the hypothesis of Theorem 5.2 and assuming that $\mathbf{m}$ is a normalized function, the following holds: If

(1) $d / l<p<\infty$ and $w \in A_{p l / d}\left(\mathbb{T}^{d}\right)$ or

(2) $1<p<(d / l)^{\prime}$ and $w^{-1 /(p-1)} \in A_{p^{\prime} l / d}\left(\mathbb{T}^{d}\right)$,

then $\left.\mathbf{m}\right|_{\mathbb{Z}^{d}} \in M_{p, w}\left(\mathbb{T}^{d}\right)$. When $l<d$ it can be taken $p=d / l$ in $(1)$ or $p=(d / l)^{\prime}$ in (2).

Moreover, if $w^{d / l} \in A_{1}\left(\mathbb{T}^{d}\right)$, then $\left.\mathbf{m}\right|_{\mathbb{Z}^{d}} \in M_{1, w}^{(\mathfrak{w})}\left(\mathbb{T}^{d}\right)$.

Proof. The result follows by applying Theorems 3.10 and 3.11 to $\mathbf{m}$.

5.2. Singular integral operators. Our second example involves the classical theory of Calderón-Zygmund singular integrals.

Definition 5.4 ([10, Definition II.5.17]). A function $K \in L_{\text {loc }}^{1}\left(\mathbb{R}^{d} \backslash\{0\}\right)$ is said to be a regular kernel if $\widehat{K} \in L^{\infty}\left(\mathbb{R}^{d}\right)$ and it satisfies

$$
\begin{array}{r}
|K(x)| \leq C|x|^{-d}, \quad \forall x \in \mathbb{R}^{d} \backslash\{0\}, \\
|K(x-y)-K(x)| \leq C|y||x|^{-d-1}, \quad|x|>2|y| .
\end{array}
$$

Corollary 5.5. Let $K$ be a regular kernel and consider for any $0<r<s<\infty$, $K_{r, s}=K \chi_{r<|x|<s}$ and $\mathbf{m}_{r, s}=\widehat{K_{r, s}}$. If $1<p<\infty$ and $w \in A_{p}\left(\mathbb{T}^{d}\right)$ there exists a constant $c$ such that

$$
\left\|\left\{\left.\mathbf{m}_{r, s}\right|_{\mathbb{Z}^{d}}\right\}_{r<s}\right\|_{M_{p, w}\left(\mathbb{T}^{d}\right)} \leq c .
$$

If $w \in A_{1}\left(\mathbb{T}^{d}\right)$, then there exists a constant $c$ such that

$$
\left\|\left\{\left.\mathbf{m}_{r, s}\right|_{\mathbb{Z}^{d}}\right\}_{r<s}\right\|_{M_{1, w}^{(\mathfrak{w})}\left(\mathbb{T}^{d}\right)} \leq c .
$$

Proof. It is easy to see that $T_{\left\{\left.\mathbf{m}_{r, s}\right|_{\mathbb{Z}^{d}}\right\}_{r<s}}^{\sharp} f(x)=T_{\left\{\left.\mathbf{m}_{r, s}\right|_{\mathbb{Z}^{d}}\right\}_{r, s \in \mathbb{Q}_{+}, r<s}}^{\sharp} f(x)$ for every $f \in P\left(\mathbb{T}^{d}\right)$. Then, the result follows by the known corresponding result for functions in $\mathbb{R}^{d}$ (see [10, Theorem IV.3.6 and V.4.11]) by applying Theorem 3.2 and its corresponding strong version. 
5.3. Bochner-Riesz partial sums. Our third application involves Bochner-Riesz partial sums. Let us recall that the Bochner-Riesz operators in $\mathbb{R}^{d}$ are defined as

$$
\left(B_{\lambda}^{r} f\right)^{\curlyvee}(\xi)=\mathbf{m}_{r}(\xi) \widehat{f}(\xi), \quad \text { where } \quad \mathbf{m}_{r}(x)=\left(1-\frac{|x|^{2}}{r^{2}}\right)_{+}^{\lambda},
$$

$t_{+}=\max (t, 0)$, and the associated maximal operator is defined by

$$
B_{\lambda}^{\sharp} f(x)=\sup _{r>0}\left|B_{\lambda}^{r} f(x)\right|
$$

for $\lambda>0$. It is known that for $\lambda>\frac{d-1}{2}, B_{\lambda}^{\sharp} f$ is pointwise majorized by the Hardy-Littlewood maximal operator; then it inherits its boundedness properties. For the critical index the following is known (S. Shi and Q. Sun [17, Theorem 1] and A. Vargas [16, Theorem 1]).

Theorem 5.6. Let $\lambda=\frac{d-1}{2}$. If $1<p<\infty$ and $w \in A_{p}\left(\mathbb{R}^{d}\right)$, then

$$
\left\|B_{\lambda}^{\sharp} f\right\|_{L^{p}\left(\mathbb{R}^{d}, w\right)} \leq C\|f\|_{L^{p}\left(\mathbb{R}^{d}, w\right)},
$$

and if $w \in A_{1}\left(\mathbb{R}^{d}\right)$, there is a constant $C$ such that for each $r>0$,

$$
\left\|B_{\lambda}^{r} f\right\|_{L^{1, \infty}\left(\mathbb{R}^{d}, w\right)} \leq C\|f\|_{L^{1}\left(\mathbb{R}^{d}, w\right)},
$$

where the constants depend only on the $A_{p}$-constant of $w$ and the dimension $d$.

Let us observe that for $\lambda=(d-1) / 2$, the kernel of the operator $B_{\lambda}^{r}$, say $K$, satisfies the size condition $|K(x)| \lesssim|x|^{-d}$, but it does not satisfy any Hörmander type condition such as (5.3) above. Then we can't apply the result obtained in the previous example.

In the periodic case, for $r>0$, the Bochner-Riesz partial sum of order $\lambda>0$ is defined for every $f \in P\left(\mathbb{T}^{d}\right)$ by

$$
S_{\lambda}^{r} f(\theta)=\sum_{|n| \leq r}\left(1-\frac{|n|^{2}}{r^{2}}\right)_{+}^{\lambda} \widehat{f}(n) e^{2 \pi i n \theta},
$$

and we denote by $S_{\lambda}^{\sharp}$ the associated maximal operator. Observe that since the function $\left(1-|x|^{2}\right)_{+}^{\lambda}$ is continuous, for every $f \in P\left(\mathbb{T}^{d}\right)$,

$$
S_{\lambda}^{\sharp} f(x)=\sup _{r \in \mathbb{Q}_{+}}\left|S_{\lambda}^{r} f(x)\right| .
$$

Then, as a consequence of our results, the following counterpart to Theorem 5.6 is obtained.

Corollary 5.7. Let $\lambda \geq \frac{d-1}{2}$. If $1<p<\infty$ and $w \in A_{p}\left(\mathbb{T}^{d}\right)$, then there exists $C>0$ such that

$$
\left\|S_{\lambda}^{\sharp} f\right\|_{L^{p}\left(\mathbb{T}^{d}, w\right)} \leq C\|f\|_{L^{p}\left(\mathbb{T}^{d}, w\right)} .
$$

If $w \in A_{1}\left(\mathbb{T}^{d}\right)$ and $\lambda=\frac{d-1}{2}$ there exists $C>0$ such that for any $r>0$,

$$
\left\|S_{\lambda}^{r} f\right\|_{L^{1, \infty}\left(\mathbb{T}^{d}, w\right)} \leq C\|f\|_{L^{1}\left(\mathbb{T}^{d}, w\right)},
$$

and if $\lambda>\frac{d-1}{2}$, there exists $C>0$ such that

$$
\left\|S_{\lambda}^{\sharp} f\right\|_{L^{1, \infty}\left(\mathbb{T}^{d}, w\right)} \leq C\|f\|_{L^{1}\left(\mathbb{T}^{d}, w\right)} .
$$


By standard arguments the theorem implies:

Corollary 5.8. Let $\lambda \geq \frac{d-1}{2}, 1 \leq p<\infty$ and $w \in A_{p}\left(\mathbb{T}^{d}\right)$. For any $f \in L^{p}\left(\mathbb{T}^{d}, w\right)$,

$$
\lim _{r \rightarrow 0^{+}} S_{\lambda}^{r} f=f
$$

where the convergence is considered in measure for $p=1$ and $\lambda=\frac{d-1}{2}$ and pointwise almost everywhere in the other cases.

For $\lambda$ below the critical index the study of the boundedness properties of the Bochner-Riesz operators constitutes an active area of research (see 9 ] for instance and the references therein). In this setting, the following is a direct consequence of [9. Theorem 5.1] (taking $u_{0}=1$ with the notation therein).

Theorem 5.9. Let $0<\lambda<(d-1) / 2$. If $w(x)=v(x)^{2 \lambda / d-1}$ with $v \in A_{2}\left(\mathbb{R}^{d}\right)$, then, for any $r>0, B_{\lambda}^{r}$ is bounded in $L^{2}\left(\mathbb{R}^{d}, w\right)$ uniformly on $r$.

Theorem 3.11 leads to obtain the following periodic counterpart result.

Corollary 5.10. Let $0<\lambda<(d-1) / 2$. If $w(x)=v(x)^{2 \lambda / d-1}$ with $v \in A_{2}\left(\mathbb{T}^{d}\right)$, then, for any $r>0, S_{\lambda}^{r}$ is bounded in $L^{2}\left(\mathbb{T}^{d}, w\right)$ uniformly on $r$.

5.4. Extension of multipliers from $L^{p}(\mathbb{T})$ to $L^{p}(\mathbb{R}, w)$. In this section we are going to show how Theorem 3.13 allows us to see the strong ties between $M_{p}(\mathbb{T})$ and a subspace of $M_{p, w}(\mathbb{R})$ for a subclass of weights in $A_{p}(\mathbb{R})$ (see Corollary $[5.12$ below).

Following M. Jodeit's ideas in [12, E. Berkson, M. Paluszyński and G. Weiss in $[5]$ gave a way to extend multipliers from $L^{p}(\mathbb{T})$ to $L^{p}(\mathbb{R}, w)$ with $w \in A_{p}(\mathbb{R})$ satisfying that there exists a constant $\rho \geq 1$ such that for each $k \in \mathbb{Z}$

$$
\rho^{-1} w(k) \leq w(x) \leq \rho w(k), \quad \text { for all } x \in[k, k+1) .
$$

These weights are said to be in $W_{p}$.

In this framework, E. Berkson, M. Paluszyński and G. Weiss proved the following result.

Theorem 5.11 ([5, Theorem 4.21]). Let $1<p<\infty, w \in W_{p}, \Psi \in M_{p, w}(\mathbb{R})$ and the support of $\Psi$ is contained in $[-1 / 2,1 / 2]$. Then, if $\left\{\phi_{n}\right\}_{n} \in M_{p}(\mathbb{T})$, we have that

$$
\mathcal{W}_{\phi, \Psi}(t)=\sum_{m \in \mathbb{Z}} \phi(m) \Psi(t-m) \in M_{p, w}(\mathbb{R})
$$

and

$$
\left\|\left\{\mathcal{W}_{\phi_{n}, \Psi}\right\}_{n}\right\|_{M_{p, w}(\mathbb{R})} \leq K_{p, w}\|\Psi\|_{M_{p, w}(\mathbb{R})}\left\|\left\{\phi_{n}\right\}_{n}\right\|_{M_{p}(\mathbb{T})} .
$$

Since $\left.\mathcal{W}_{\phi_{n}, \Psi}\right|_{\mathbb{Z}}=\left.\Psi(0) \phi_{n}\right|_{\mathbb{Z}}$, a direct consequence of Theorem 3.13 with $u=1$ and $v=w$ is that the converse of Theorem 5.11 also holds:

Corollary 5.12. Let $\left\{\phi_{n}\right\}_{n} \subset \ell^{\infty}(\mathbb{Z})$. Then, under the hypothesis of Theorem 5.11, we have that if $\Psi(0) \neq 0$,

$$
\left\|\left\{\mathcal{W}_{\phi_{n}, \Psi}\right\}_{n}\right\|_{M_{p, w}(\mathbb{R})}<+\infty \text { if and only if }\left\|\left\{\phi_{n}\right\}_{n}\right\|_{M_{p}(\mathbb{T})}<+\infty \text {. }
$$


Moreover,

$$
\frac{C_{p, w}\left\|\left\{\phi_{n}\right\}_{n}\right\|_{M_{p}(\mathbb{T})}}{|\Psi(0)|} \leq\left\|\left\{\mathcal{W}_{\phi_{n}, \Psi}\right\}_{n}\right\|_{M_{p, w}(\mathbb{R})} \leq K_{p, w}\|\Psi\|_{M_{p, w}(\mathbb{R})}\left\|\left\{\phi_{n}\right\}_{n}\right\|_{M_{p}(\mathbb{T})} .
$$

Observation 5.13. In the particular case of a single multiplier, inequality (5.5) yields that, for any $w \in W_{p}$, the map $\phi \mapsto \mathcal{W}_{\phi, \Psi}$ induces an isomorphism between $M_{p}(\mathbb{T})$ and a subspace of $M_{p, w}(\mathbb{R})$. This result is a one dimensional weighted generalization of the unweighted result in [12, p. 225] for $\Psi$ the characteristic function of the interval $[-1 / 2,1 / 2)$.

\section{ACKNOWLEDGEMEnTS}

We would like to thank the referee for some valuable comments which have improved the final version of this work.

\section{REFERENCES}

[1] K. Andersen and P. Mohanty, Restriction and extension of Fourier multipliers between weighted $L^{p}$ spaces on $\mathbb{R}^{n}$ and $\mathbb{T}^{n}$, Proc. Amer. Math. Soc. 137 (2009), no. 5, 1689-1697. MR 2470827 (2009m:42012)

[2] N. Asmar, E. Berkson, and J. Bourgain, Restrictions from $\mathbb{R}^{n}$ to $\mathbb{Z}^{n}$ of weak type $(1,1)$ multipliers, Studia Math. 108 (1994), no. 3, 291-299. MR.1259281 (95b:42015)

[3] N. Asmar, E. Berkson, and T. A. Gillespie, Maximal estimates on measure spaces for weaktype multipliers, J. Geom. Anal. 5 (1995), no. 2, 167-179. MR1341028 (96m:43006)

[4] E. Berkson and T. A. Gillespie, On restrictions of multipliers in weighted settings, Indiana Univ. Math. J. 52 (2003), no. 4, 927-961. MR.2001939 (2005i:43006)

[5] E. Berkson, M. Paluszyński, and G. Weiss, Transference couples and weighted maximal estimates, Harmonic analysis and partial differential equations (Chicago, IL, 1996), 1999, pp. 4967. MR 1743855 (2001d:47045)

[6] M. J. Carro and S. Rodríguez, New results on restriction of Fourier multipliers, Mathematische Zeitschrift 265 (2010), no. 2, 417-435. MR2609318 (2011c:42029)

[7] _ Transference results on weighted Lebesgue spaces, Proc. Roy. Soc. Edinburgh Sect. A 138 (2008), no. 2, 239-263. MR2406690 (2009d:43009)

[8] K. De Leeuw, On L $L_{p}$ multipliers, Ann. of Math. (2) 81 (1965), 364-379. MR0174937(30:5127)

[9] J. Duoandikoetxea, A. Moyua, O. Oruetxebarria, and E. Seijo, Radial Ap weights with applications to the disc multiplier and the Bochner-Riesz operators, Indiana Univ. Math. J. 57 (2008), no. 3, 1261-1281. MR2429092 (2009d:42034)

[10] J. García-Cuerva and J. L. Rubio de Francia, Weighted norm inequalities and related topics, North-Holland Mathematics Studies, vol. 116, North-Holland Publishing Co., Amsterdam, 1985. Notas de Matemática [Mathematical Notes], 104. MR807149 (87d:42023)

[11] R. Hunt, B. Muckenhoupt, and R. Wheeden, Weighted norm inequalities for the conjugate function and Hilbert transform, Trans. Amer. Math. Soc. 176 (1973), 227-251. MR0312139 $(47: 701)$

[12] M. Jodeit, Jr., Restrictions and extensions of Fourier multipliers, Studia Math. 34 (1970), 215-226. MR0262771 (41:7376)

[13] C. E. Kenig and P. A. Tomas, Maximal operators defined by Fourier multipliers, Studia Math. 68 (1980), no. 1, 79-83. MR583403 (82c:42016)

[14] D. S. Kurtz and R. L. Wheeden, Results on weighted norm inequalities for multipliers, Trans. Amer. Math. Soc. 255 (1979), 343-362. MR.542885 (81j:42021)

[15] J. A. Raposo, Weak type $(1,1)$ multipliers on LCA groups, Studia Math. 122 (1997), no. 2, 123-130. MR:1432163 (98b:43011)

[16] X. L. Shi and Q. Y. Sun, Weighted norm inequalities for Bochner-Riesz operators and singular integral operators, Proc. Amer. Math. Soc. 116 (1992), no. 3, 665-673. MR1136237 (93a:42009) 
[17] A. M. Vargas, Weighted weak type $(1,1)$ bounds for rough operators, J. London Math. Soc. (2) 54 (1996), no. 2, 297-310. MR1405057 (98g:42027)

Departament de Matemàtica Aplicada i Anàlisi, Universitat de Barcelona, 08007 Barcelona, Spain

E-mail address: carro@ub.edu

URL: http://garf.ub.edu

Department of Mathematics, Uppsala University, Upssala, SE 75106, Sweden

E-mail address: salvador@math.uu.se

$U R L$ : www.math.uu.se/ salvador 